\title{
Policy initiative to improve access to psychological services for people with affective and anxiety disorders: population-level analysis ${ }^{\dagger}$
}

\author{
Meredith G. Harris, Philip M. Burgess, Jane E. Pirkis, Tim N. Slade and Harvey A. Whiteford
}

\section{Background}

In 2006, Australia introduced new publicly funded psychological services for people with affective and anxiety disorders (the Better Access programme). Despite massive uptake, it has been suggested that Better Access is selectively treating socioeconomically advantaged people, including some who do not warrant treatment, and people already receiving equivalent services.

\section{Aims}

To explore potential disparities in Better Access treatment using epidemiological data from the 2007 National Survey of Mental Health and Wellbeing.

\section{Method}

Logistic regression analyses examined patterns and correlates of service use in two populations: people who used the new psychological services in the previous 12 months; and people with any ICD-10 12-month affective and anxiety disorder, regardless of service use.

\section{Results}

Most (93.2\%) Better Access psychological services users had a 12-month ICD-10 mental disorder or another indicator of treatment need. Better Access users without affective or anxiety disorders were not more socioeconomically advantaged, and received less treatment than those with these disorders. Among the population with affective or anxiety disorders, non-service users were less likely to have a severe disorder and more likely to have anxiety disorder, without a comorbid affective disorder, than Better Access users. Better Access users comprised more new allied healthcare recipients than other service users. A substantial minority of non-service users (13.5\%) had severe disorders, but most did not perceive a need for treatment.

\section{Conclusions}

Better Access does not appear to be overservicing individuals without potential need or contributing to social inequalities in mental healthcare. It appears to be reaching people who have not previously received psychological care. Treatment rates could be improved for some people with anxiety disorders.

\section{Declaration of interest}

None.
Improving treatment rates for common mental disorders is a challenge for all governments. Australia has instituted a number of primary mental healthcare reforms in response to the high level of burden and low levels of treatment associated with affective and anxiety disorders. ${ }^{1,2}$ The most recent and largest of these is the Better Access to Psychiatrists, Psychologists and General Practitioners through the Medicare Benefits Schedule (Better Access) initiative, which was introduced in November $2006^{3}$ and is similar in scope and intent to the UK's Improving Access to Psychological Therapies initiative. ${ }^{4}$ Under Better Access, a range of new psychological services was made available through Medicare, Australia's universal health insurance system. Medicare now covers all or part of the costs of a capped number of psychological services delivered by psychologists and selected social workers and occupational therapists in the community, on referral from a general practitioner (GP), psychiatrist or paediatrician. Previously, these costs would have been borne by the consumer, either directly or through private health insurance. Uptake has been substantial - more than 1.2 million Better Access psychological services (59 per 1000 total population) were provided by allied health professionals in the first year, ${ }^{5}$ with the number almost doubling (105 per 1000) in the second year ${ }^{6}$ - suggesting that the initiative is addressing a previously unmet need. A potential risk of demand-driven programmes such as Better Access is that they may create, or widen existing, disparities in mental health service use. Limited population-level evaluation

†See editorial, pp. 91-92, this issue. of the programme has been conducted to date. However, it has been suggested that the initiative is providing services to a number of people who may not have affective or anxiety disorders, and who live in affluent and urban areas (where most allied health professionals practice), whereas at-risk groups (e.g. young people, people in rural areas or poorer urban areas, people with low income) with legitimate need may be missing out. A further concern is that, rather than providing access to services for previously untreated individuals, the initiative is providing funding for people already receiving care from psychologists or other allied health providers. ${ }^{7-10}$ These criticisms are based on evidence drawn from analyses of Medicare claims data, however the person-level data collected by Medicare captures only limited demographic data. This makes it difficult to profile service recipients, particularly with respect to clinical characteristics. This paper explores whether Better Access is contributing to mental healthcare disparities using epidemiological data from the 2007 National Survey of Mental Health and Wellbeing. The survey collected detailed information on sociodemographic and clinical characteristics as well as service use for mental health problems.

\section{Method}

\section{Research questions}

Our research questions were aligned with the nominated concerns about Better Access. Two populations were of interest for the current study: people who had consulted a Medicare-funded psychologist or other allied health specialist for a mental health problem in the past 12 months (Better Access psychological 
services users); and a community sample of people with 12-month affective and anxiety disorders regardless of service use, who are the primary target of Better Access. Among the Better Access psychological service users, we sought to determine: is there evidence of overservicing of people without potential need; and are users without affective or anxiety disorders more socioeconomically advantaged than those with these disorders? Among the community sample with any 12-month ICD-10 affective or anxiety disorder we sought to determine: are there any population subgroups who appear to be missing out on Better Access psychological services; is Better Access selectively providing services to people already receiving equivalent treatment; and is there perceived need for treatment among people who do not receive services?

\section{Sampling and procedure}

The 2007 National Survey of Mental Health and Wellbeing was conducted by the Australian Bureau of Statistics between August and December 2007. The population in scope was usual residents of private dwellings across Australia, aged 16-85 years. A random sample of private dwellings was identified using a stratified, multistage area probability sampling technique. Information about household composition was obtained and an algorithm developed by the Australian Bureau of Statistics used to randomly select one person in the household aged 16-85 years to be interviewed. Younger people (16-24 years) and older people (65-85 years) were oversampled in order to improve the reliability of estimates for these groups. Of 14805 eligible households identified, interviews were completed with $8841(60 \%)$ of respondents. The survey methodology is described in detail elsewhere. ${ }^{11,12}$

\section{Defining the populations of interest}

\section{Diagnostic assessment}

The current version of the World Mental Health Composite International Diagnostic Interview (CIDI 3.0) ${ }^{13}$ was used to assess the prevalence of lifetime diagnoses according to ICD-10 14 criteria. Questions about symptoms experienced during the 12 months prior to interview were combined with lifetime diagnosis information to determine 12-month prevalence. The survey assessed the following classes of mental disorder: affective disorders - depression, dysthymia and bipolar affective disorder; anxiety disorders - panic disorder, agoraphobia, social phobia, generalised anxiety disorder, obsessive-compulsive disorder and post-traumatic stress disorder; and substance use disorders harmful use and dependence syndrome separately for alcohol, opioids, cannabinoids, sedatives and stimulants.

\section{Measurement of service use for mental health problems}

The service use module of the 2007 National Survey of Mental Health and Wellbeing gathered information about respondents' 12-month and lifetime use of services for mental health problems. Respondents were asked whether they had consulted any of the following professional groups for mental health problems: GPs; psychiatrists; psychologists; mental health nurses; other mental health specialists (including social workers, occupational therapists and counsellors); other specialist doctors or surgeons; other professionals providing general services; and complementary/ alternative therapists. For each type of professional consulted, respondents were asked whether they had consulted within the past 12 months for mental health problems, the number of consultations within the past 12 months for mental health problems, how these services were paid for and their age at first ever consultation. Payment source was classified into non-mutually exclusive categories: public hospital out-patients, public community health services or public community mental health services; paid or refunded in full by Medicare; paid or refunded in part by Medicare; paid or refunded in full by private insurance; paid or refunded in part by private insurance; paid in full by respondent, a friend or family member; and paid in part by respondent, a friend or family member. Age at first ever consultation was coded by the Australian Bureau of Statistics into ranges (psychologists: < 16, 16-17, 18-21, 22-25, 26-29, 30-32, $33-37,38-42,43-47,48-54,55$ years and over; other mental health specialists, $<18,18-23,24-29,30-36,37-50,51$ years and over). Respondents were also asked whether they had been hospitalised for a mental health problem, or had used selfmanagement strategies including internet support groups or chat rooms, self-help groups and telephone counselling.

\section{Better Access psychological services users}

To identify likely Better Access psychological services users, we selected respondents who had consulted a psychologist or other mental health specialist for a mental health problem in the past 12 months, and who reported that these services were paid for in full or in part by Medicare. We considered this strategy appropriate for two reasons. First, although psychologists, social workers and occupational therapists are able to claim Medicare subsidies for a limited range of non-Better Access mental health and other services, these represented only $2 \%$ of all Medicaresubsidised mental health services provided by these groups in 2007. Second, although the other mental health specialist group included counsellors, counsellors are not able to claim Medicare subsidies.

\section{Identifying disability, clinical status, treatment need and sociodemographic characteristics}

\section{Disability measures}

Disability was assessed using the 12-item version of the World Health Organization Disability Assessment Schedule (WHODAS-II), ${ }^{15}$ which asks respondents how much difficulty they had in the preceding 30 days in performing a range of activities. A score at or above the seventy-fifth percentile indicates a high level of disability. Disability was also assessed using a measure of 'days out of role'. Respondents were asked how many days in the previous 30 they were totally unable to perform their normal activities, and how many days they had to cut down what did they did because of health problems. Total 'days out of role' was calculated as the weighted sum of total plus partial days out of role.

\section{Clinical measures}

Respondents with a 12-month ICD-10 disorder were classified into one of three severity categories - mild, moderate or severe - using criteria adapted from the World Mental Health Survey Initiative. ${ }^{11,12}$ Psychological distress during the 30 days prior to interview was assessed using the Kessler Psychological Distress Scale (K10). ${ }^{16}$ Respondents were also asked whether they had any of the following chronic physical conditions: diabetes, asthma, coronary heart disease, stroke, cancer and arthritis.

\section{Treatment need}

Respondents were classified on a gradient of potential need for treatment, using criteria adapted from Druss and colleagues. ${ }^{17}$ The gradient comprised three hierarchically ordered groups: any 12-month ICD-10 disorder; one or more of the following indicators of potential need - a lifetime ICD-10 disorder, 12-month symptoms (but no lifetime diagnosis) for at least one disorder, or 
lifetime hospitalisation for a mental health problem; and none of the preceding indicators of potential treatment need. The Perceived Need for Care Questionnaire (PNCQ) ${ }^{18}$ assessed the extent to which identified treatment needs were met among respondents who had received services in the past 12 months. It was also used to assess perceived need for treatment and reasons for not receiving treatment among those who had not received services.

\section{Sociodemographic characteristics}

Sociodemographic information included respondents' age, gender, marital status, labour force status, highest level of education attained, country of birth, language spoken at home, area of residence and household income. Respondents were classified into deciles on the Index of Relative Socioeconomic Disadvantage, ${ }^{19}$ based on information about the geographic location of their household. The Index of Relative Socioeconomic Disadvantage provides a continuum of advantage (high values) to disadvantage (low values).

\section{Data analysis}

For analyses involving the Better Access psychological services users, we used binomial logistic regressions analyses to compare individuals with and without a 12-month ICD-10 affective or anxiety disorder on a range of sociodemographic, mental health service use and treatment need measures. For analyses involving the sample with any ICD-10 12-month affective or anxiety disorder, we used multinomial logistic regression analyses to compare three service use groups on various sociodemographic and clinical factors: Better Access psychological service users (those who received Medicare-subsidised allied health services, with or without other mental health service use); other mental health service users; and non-service users. Univariate analyses first assessed the unadjusted associations between each predictor and the dependent variable. In order to identify the most parsimonious model for predicting membership in service use groups, a multivariate analysis was then undertaken. Variables that failed to make a significant independent contribution to model fit were removed from analysis, based on the Wald chi-squared statistic $(P<0.05)$. Multicollinearity was assessed by inspecting correlations between variables, tolerance and variance inflation factors. Binomial logistic regression analyses were also used to compare Better Access and other mental health service users on interventions received and proportion of potentially new cases to treatment.

Data were made available by the Australian Bureau of Statistics via the Basic Confidentialised Unit Record File (April 2009 version). Analyses were conducted using STATA version 10.1 for Windows. Standard errors (s.e.) and 95\% confidence intervals were calculated using jackknife repeated replication, which provides unbiased estimates of the sampling error arising from complex sample selection procedures. Estimates with relative standard errors between $25 \%$ and $50 \%$, indicating some limitations to reliability, were identified as per Australian Bureau of Statistics practice. ${ }^{20}$ Statistical tests were two-tailed. $P$-values less than 0.05 were considered statistically significant.

\section{Results}

\section{Overservicing}

We looked at whether there was evidence that Better Access is overservicing people without potential need. Figure 1 shows the relative size of and overlap between the populations of Better
Access psychological services users and the community sample with any 12-month affective or anxiety disorder. Of the total sample of 8841 respondents, 175 had used Better Access psychological services in the 12 months prior to interview, which roughly corresponds to the first year of the programme. This equates to an estimated population count of 290140 persons, or $1.8 \%$ of Australian adults aged 16-85 years. Three-quarters $(74.7 \%$, s.e. $=5.4)$ received these services exclusively from a psychologist, a fifth from other mental health specialists only $(21.1 \%$, s.e. $=5.1)$, and less than one in twenty from both a psychologist and another mental health specialist $(4.3 \%$, s.e. $=2.6)$. Better Access users represented $37.3 \%$ (s.e. $=3.5$ ) of all adult Australians who received services from these providers.

Among Better Access psychological services users, 50.1\% (s.e. $=6.2$ ) had a 12 -month ICD-10 affective disorder and $60.9 \%$ $($ s.e. $=5.4)$ had a 12 -month ICD-10 anxiety disorder (categories not mutually exclusive). An estimated $14.2 \%$ (s.e.=4.9) had a 12-month ICD-10 substance use disorder, however the majority of these $(78.4 \%$, s.e. $=9.5)$ were comorbid with 12-month ICD-10 affective or anxiety disorders. Almost one in five $(18.3 \%$, s.e. $=3.3)$ Better Access users did not have a 12-month ICD-10 disorder.

The majority of Better Access users had a potential need for treatment: $81.7 \%$ (s.e. $=3.3$ ) had a 12-month ICD-10 affective, anxiety or substance use disorder; $11.5 \%($ s.e. $=2.1)$ had at least one other indicator of potential need (lifetime disorder, 12-month symptoms or lifetime hospitalisation for a mental disorder); and $6.8 \%$ (s.e. $=2.2$ ) had no indicator of potential need.

\section{Socioeconomic profile}

We examined whether Better Access users without a 12-month ICD-10 affective or anxiety disorder $(21.3 \%$, s.e. $=3.5)$ had a different socioeconomic profile than users with these disorders $(78.7 \%$, s.e. $=3.5)$. There were no statistically significant differences between the groups (Table 1).

A greater proportion of Better Access users with 12-month ICD-10 affective or anxiety disorders received 10 or more

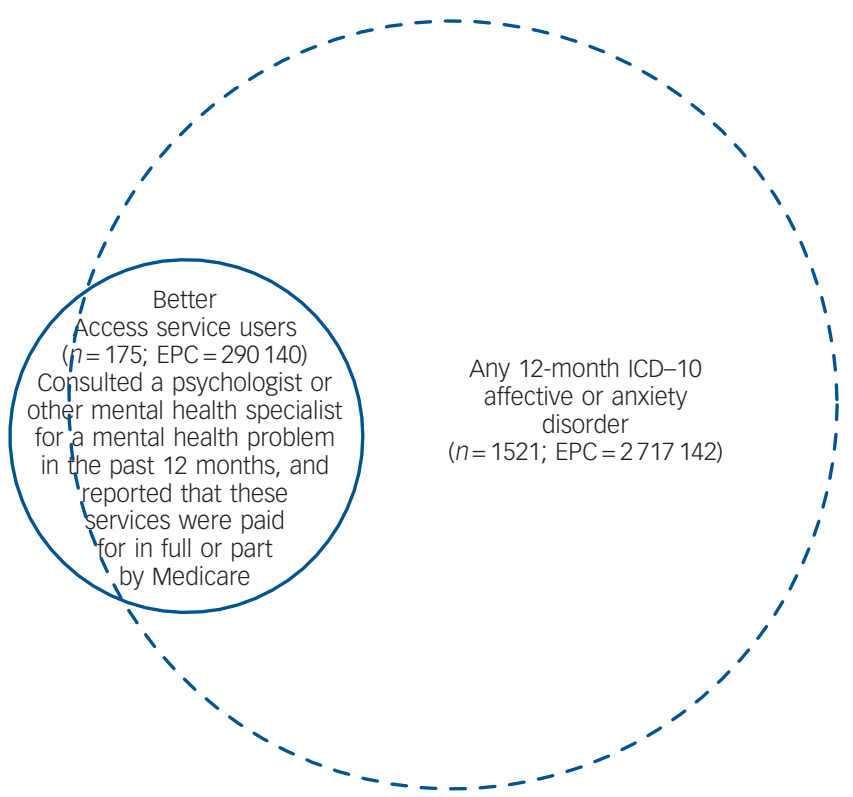

Fig. 1 Overlap between Better Access psychological services users and the community sample with any 12-month ICD-10 affective or anxiety disorder

$n$, unweighted number of respondents; EPC, estimated population count. 
Table 1 Sociodemographic correlates of 12-month ICD-10 affective or anxiety disorder status among Better Access psychologica services users ${ }^{\mathrm{a}}$

\begin{tabular}{|c|c|c|c|c|c|c|c|}
\hline & \multicolumn{2}{|c|}{$\begin{array}{l}\text { No 12-month ICD-10 affective } \\
\text { or anxiety disorder }(N=51)^{\mathrm{b}}\end{array}$} & \multicolumn{2}{|c|}{$\begin{array}{l}\text { Any } 12 \text {-month ICD-10 affective } \\
\text { or anxiety disorder }(N=124)^{\mathrm{b}}\end{array}$} & \multirow{2}{*}{\multicolumn{2}{|c|}{$\mathrm{OR}^{\mathrm{C}}(95 \% \mathrm{Cl})$}} & \multirow[b]{2}{*}{$P$} \\
\hline & $n^{\mathrm{b}}$ & $\%$ (s.e.) & $n^{\mathrm{b}}$ & $\%$ (s.e.) & & & \\
\hline Female & 36 & $64.5(8.6)$ & 94 & $67.1(6.1)$ & 1.1 & $(0.4-3.0)$ & 0.813 \\
\hline \multicolumn{8}{|l|}{ Age group } \\
\hline $16-34$ years & 14 & $31.7(7.7)$ & 47 & $40.5(6.3)$ & 1.0 & & \\
\hline $35-54$ years & 21 & $40.6(7.0)$ & 54 & $45.4(6.7)$ & 0.9 & (0.3-2.2) & 0.777 \\
\hline $55-85$ years & 16 & $27.7^{\mathrm{d}}(7.4)$ & 23 & $14.1^{\mathrm{d}}(3.6)$ & 0.4 & $(0.1-1.2)$ & 0.108 \\
\hline Married & 16 & $34.9(7.7)$ & 33 & $36.6(7.5)$ & 1.1 & $(0.4-3.1)$ & 0.889 \\
\hline Born in Australia & 37 & $71.0(7.6)$ & 102 & $85.3^{d}(3.9)$ & 2.4 & $(0.8-7.1)$ & 0.121 \\
\hline Lives in major urban area & 33 & $68.1(8.1)$ & 81 & $76.3^{\mathrm{d}}(4.3)$ & 1.5 & $(0.6-3.7)$ & 0.365 \\
\hline Least socioeconomic disadvantage (IRSED deciles 7-10) & 23 & $41.6(8.3)$ & 57 & $40.2(6.1)$ & 0.9 & $(0.4-2.3)$ & 0.893 \\
\hline Post-school qualification & 35 & $62.1(8.5)$ & 71 & $53.5(6.7)$ & 0.7 & $(0.3-1.6)$ & 0.395 \\
\hline Employed & 33 & $58.0(7.1)$ & 69 & $52.1(6.9)$ & 0.8 & $(0.4-1.7)$ & 0.524 \\
\hline High level of income (household income deciles 7-10) & 17 & $35.2(8.5)$ & 31 & $26.0(6.4)$ & 0.6 & $(0.2-1.9)$ & 0.421 \\
\hline \multicolumn{8}{|c|}{$\begin{array}{l}\text { IRSED, Index of Relative Socioeconomic Disadvantage. } \\
\text { a. Better Access psychological services users consulted a psychologist or other mental health specialist for a mental health problem in the past } 12 \text { months, and reported that thes } \\
\text { services were paid for in full or in part by Medicare. } \\
\text { b. } N \text { and } n \text {, unweighted number of respondents. } \\
\text { C. Odds ratios (ORS) are from univariate logistic regression analyses with no } 12 \text {-month affective or anxiety disorder as the reference group. } \\
\text { d. The relative standard error for this estimate, or its reference category, represents } 25-30 \% \text { of the estimate value. } \\
\text { e. } N=48 \text { and } N=109 \text { for this variable. }\end{array}$} \\
\hline
\end{tabular}

consultations for a mental health problem in the past year, but there were no differences in types of help received or whether perceived needs for treatment were met (Table 2).

\section{Underservicing}

Among the community sample with any ICD-10 12-month affective or anxiety disorder, we looked at whether there were any population subgroups who appear to be missing out on Better Access psychological services. Figure 2 shows the rates of service use for a mental health problem in the past 12 months among people with and without 12-month ICD-10 affective or anxiety disorders. Notably, $8.4 \%$ of people with these disorders actually used Better Access psychological services, and 21.5\% of those who used any services for a mental health problem in the past 12 months used Better Access.

The remaining analyses focused on the community sample with any 12-month ICD-10 affective or anxiety disorder. Tables 3 and 4 summarise the associations between sociodemographic, clinical and disability measures and membership in three different service use groups - Better Access, other service use and no service use. There were no differences between Better Access psychological service users and other service users on any of these measures. There were, however, a number of differences between the Better Access and non-service user groups. Non-service users had twice the odds of being employed compared with the Better Access group (Table 3). They had 3.6 times the odds of having an anxiety disorder as their only 12-month mental disorder but only a

Table 2 Treatment correlates of 12-month ICD-10 affective or anxiety disorder status among Better Access psychological services users ${ }^{\mathrm{a}}$

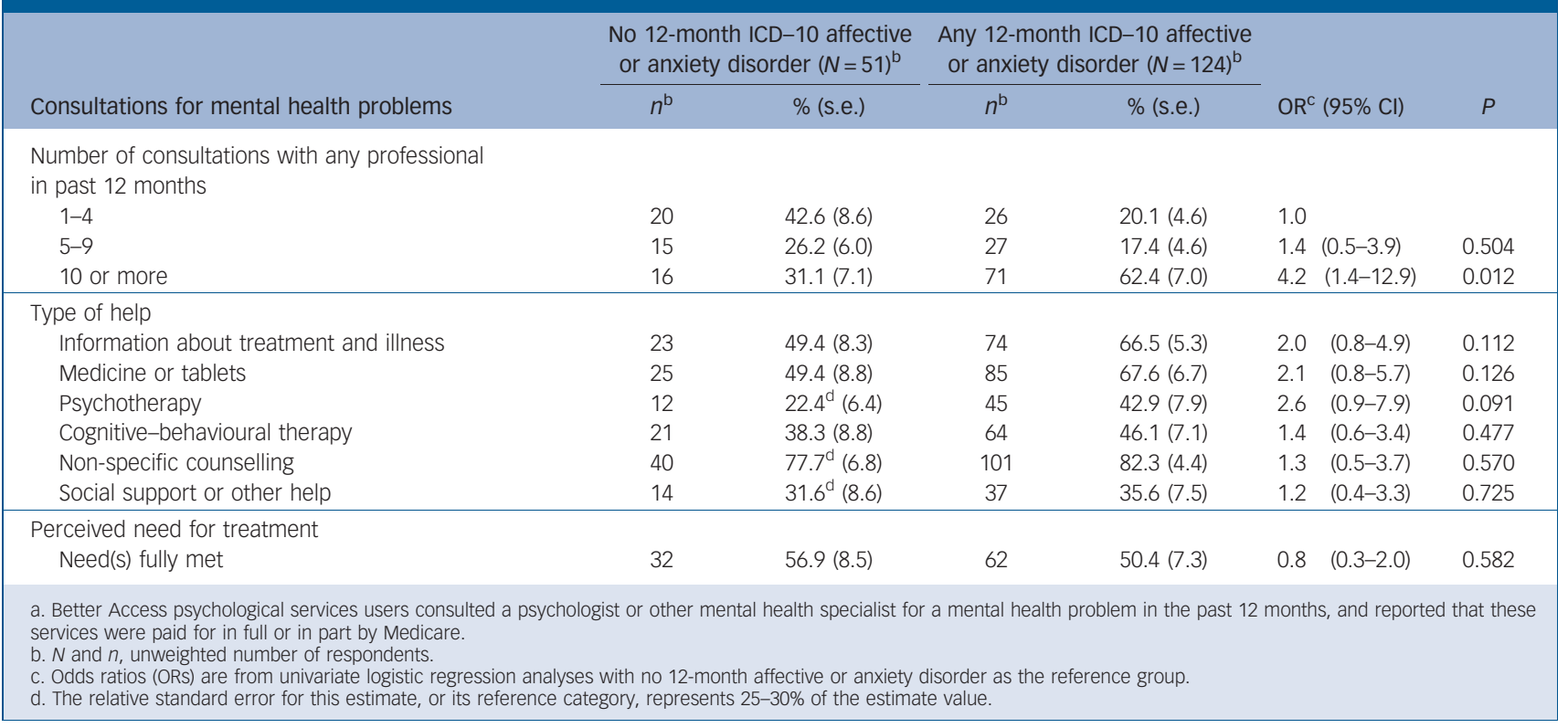




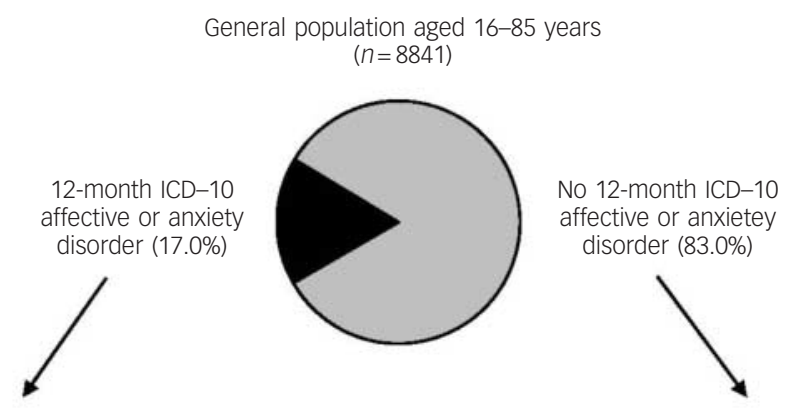

Service use by people with 12-month ICD-10 affective or anxiety disorders $(n=1521)$
Service use by people with no 12-month ICD-10 affective or anxiety disorder $(n=7320)$

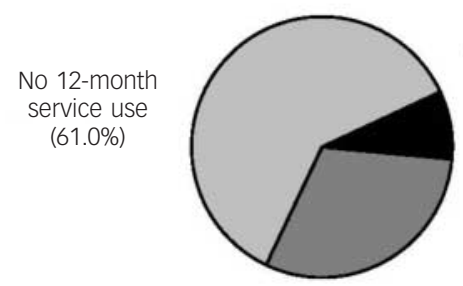

12-month use of Better Access psychological services $(8.4 \%)$

12-month use of other services (30.6\%)

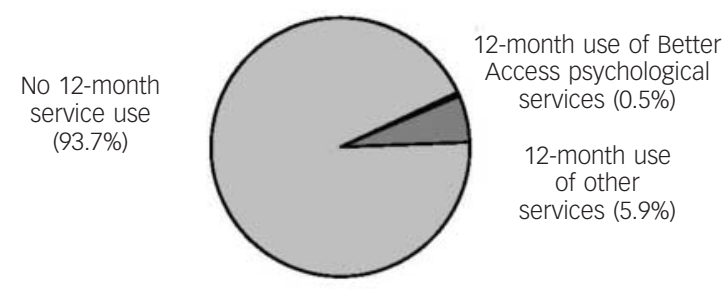

Fig. 2 Use of services for a mental health problem in the past 12 months among people with and without a 12-month ICD-10 affective or anxiety disorder.

$n$, unweighted number of respondents. Percentages may not sum to 100 because of rounding.

quarter of the odds of having a 12-month affective disorder, either alone or in combination with another disorder. Non-service use was also negatively associated with severity, days out of role and psychological distress (Table 4).

The multivariate multinomial analysis showed, however, that two variables were the key determinants of whether a person with 12-month affective or anxiety disorder was a non-service user, compared with being a Better Access user. These were: lower odds of having a severe disorder (adjusted odds ratio $(\mathrm{OR})=0.3,95 \%$ CI $0.2-0.6, P=0.002$ ); and lower odds of having a 12-month ICD-10 affective disorder, either alone (adjusted OR $=0.3,95 \%$ CI $0.1-0.8, P=0.016$ ), or in combination with another disorder (adjusted OR $=0.3,95 \%$ CI $0.1-0.6, P=0.001$ ), compared with not having an affective disorder. Because the sample for this analysis comprises individuals with an affective or anxiety disorder, respondents in the reference category 'no affective disorder' are those who had an anxiety disorder that is not comorbid with an affective disorder.

We were also interested in whether service use patterns differed between the Better Access and other service user groups. Binomial logistic regression analyses showed a monotonic increasing association between Better Access service use and number of mental health consultations in the past 12 months. Better Access users also had twice the odds of reporting receipt of information about illness and treatment and almost four times the odds of reporting receipt of non-specific counselling than other service users. There were no significant differences in self-reported rates of receipt of other interventions, including cognitive-behavioural therapy (CBT) (Table 5).

\section{New to treatment}

Ideally, the question of whether Better Access is selectively providing services to people already receiving equivalent treatment would be examined by determining the proportion of Better Access service users who had seen a psychologist or other mental health specialist for the first time in the past year. However, as data on age at first ever consultation was only available in ranges, we could not calculate the exact period elapsed since first consultation. Hence, we took the following approach. Current age was recoded into age ranges equivalent to those used to report age at first consultation, separately for each type of professional consulted because the available age ranges differed by provider type. Individuals whose age ranges matched were deemed to be potentially 'new' cases. We then compared the proportion of potentially new cases in the Better Access group with the other service user group. Among individuals who had consulted a psychologist in the past 12 months $(n=336)$, there was a significantly greater proportion of potentially new cases in the Better Access group $(54.4 \%$, s.e. $=7.5)$ than in the other service user group $(30.5 \%$, s.e. $=4.3, \quad \mathrm{OR}=2.7,95 \%$ CI 1.3-5.9, $P=0.011)$, but no difference in the subsample $(n=177)$ who had seen another mental health specialist $(48.0 \%($ s.e. $=13.6) v$. $44.2 \%$ (s.e. $=6.3$ ); $\mathrm{OR}=1.2,95 \%$ CI $0.3-4.1, P=0.812$ ). When results for psychologists and other mental health specialists were combined $(n=412)$, we found a significantly greater proportion of potentially new cases in the Better Access group compared with the other service users $(62.3 \%($ s.e. $=6.7)$ v. $38.7 \%$ (s.e. $=3.5)$, $\mathrm{OR}=2.6,95 \%$ CI $1.4-4.9, P=0.003)$.

\section{Perceived need for treatment among non-service users}

One in six people with 12-month ICD-10 affective and anxiety disorders who did not use services reported a perceived need for treatment (Table 6). A greater proportion of those with severe disorder had a perceived need $(36.1 \%$, s.e. $=6.8)$, compared with those with mild or moderate disorders $(9.2 \%$, s.e. $=1.7$, $\mathrm{OR}=5.6,95 \%$ CI $2.7-11.7, P<0.001)$.

Among those who identified any perceived need, the most common need was for 'talking' therapies. When asked their reasons for not seeking help for their need(s), the most common response selected by respondents was that they preferred to 


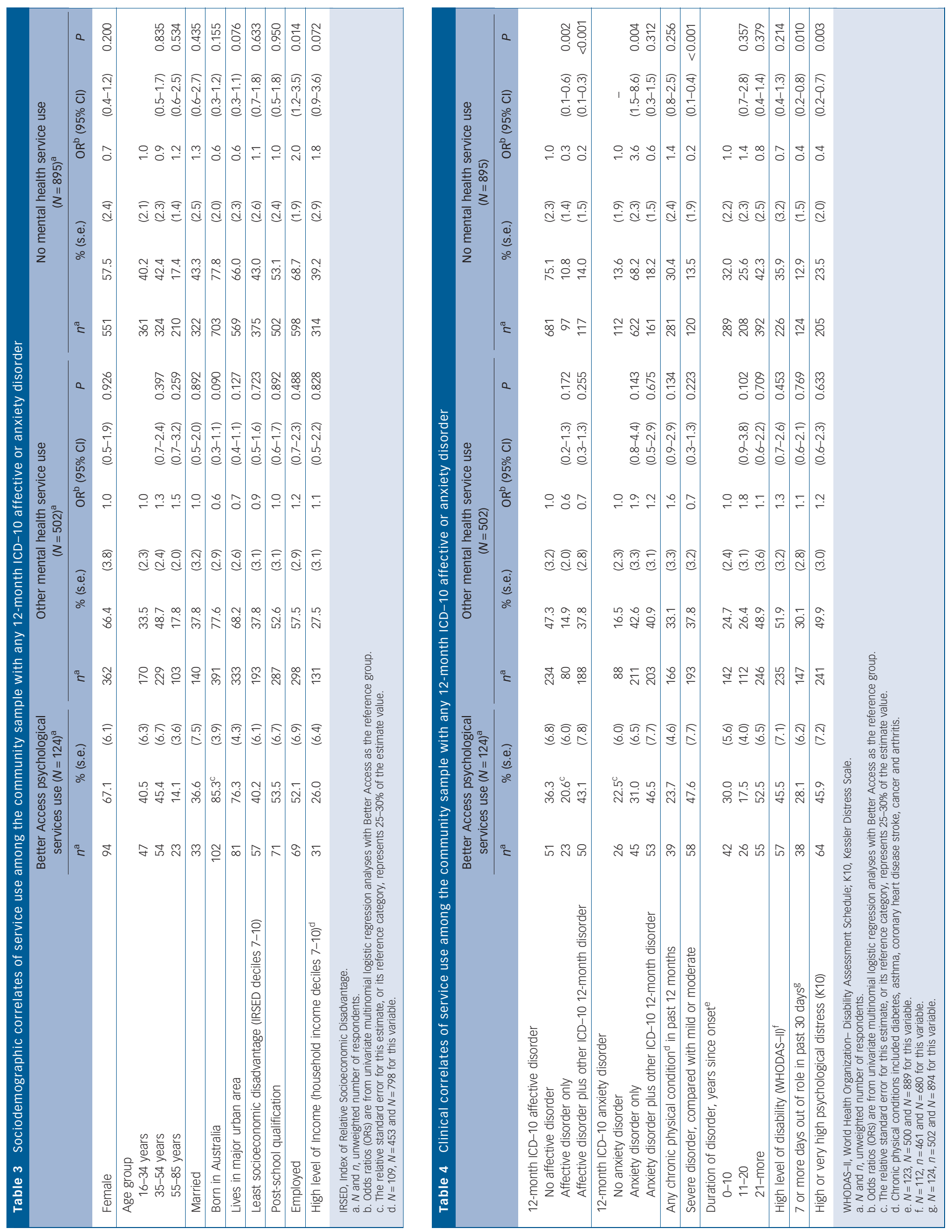




\begin{tabular}{|c|c|c|c|c|c|c|c|c|c|}
\hline & \multicolumn{3}{|c|}{$\begin{array}{l}\text { Other mental health } \\
\text { service use }(N=502)\end{array}$} & \multicolumn{3}{|c|}{$\begin{array}{l}\text { Better Access psychological } \\
\text { services use }(N=124)\end{array}$} & \multirow{2}{*}{\multicolumn{2}{|c|}{$\mathrm{OR}^{\mathrm{b}}(95 \% \mathrm{Cl})$}} & \multirow[b]{2}{*}{$P$} \\
\hline & $n^{a}$ & & s.e.) & $n^{\mathrm{a}}$ & & s.e.) & & & \\
\hline \multicolumn{10}{|l|}{$\begin{array}{l}\text { Consultations for mental health problems } \\
\text { Number of consultations with any professional } \\
\text { in past } 12 \text { months }^{c}\end{array}$} \\
\hline $1-4$ & 261 & 52.4 & (3.1) & 26 & 20.1 & (4.6) & 1.0 & - & \\
\hline $5-9$ & 94 & 17.6 & (2.3) & 27 & 17.4 & $(4.6)$ & 2.6 & $(1.2-5.6)$ & 0.017 \\
\hline 10 or more & 143 & 29.9 & (3.1) & 71 & 62.4 & (7.0) & 5.4 & $(2.7-11.0)$ & $<0.001$ \\
\hline Consulted GP for mental health problem in past 12 months $^{d}$ & 369 & 73.9 & $(2.2)$ & 95 & 73.1 & (5.6) & 1.0 & $(0.5-1.8)$ & 0.892 \\
\hline \multicolumn{10}{|l|}{ Intervention type } \\
\hline Information about illness & 239 & 48.2 & (3.1) & 74 & 66.5 & (5.3) & 2.1 & $(1.2-3.7)$ & 0.008 \\
\hline Medicine or tablets & 335 & 66.9 & (3.0) & 85 & 67.6 & (6.7) & 1.0 & $(0.5-1.9)$ & 0.924 \\
\hline Psychotherapy & 143 & 29.4 & (3.0) & 45 & 42.9 & $(7.9)$ & 1.8 & $(0.9-3.8)$ & 0.121 \\
\hline Cognitive behavioural therapy & 154 & 32.5 & (3.7) & 64 & 46.1 & $(7.1)$ & 1.8 & $(0.9-3.5)$ & 0.095 \\
\hline Non-specific counselling & 274 & 55.0 & (2.5) & 101 & 82.3 & $(4.5)$ & 3.8 & $(1.8-8.0)$ & 0.001 \\
\hline Social support or other help & 126 & 26.1 & (3.0) & 37 & 35.6 & $(7.5)$ & 1.6 & $(0.8-3.0)$ & 0.168 \\
\hline Received medication appropriate for affective/anxiety disorder & 248 & 51.2 & (3.4) & 68 & 51.7 & (7.0) & 1.0 & $(0.6-1.8)$ & 0.942 \\
\hline \multicolumn{10}{|l|}{ Needs for care met } \\
\hline Need(s) fully met & 235 & 43.4 & (3.0) & 62 & 50.4 & (7.3) & 1.3 & $(0.7-2.6)$ & 0.401 \\
\hline \multicolumn{10}{|c|}{$\begin{array}{l}\text { GP, general practitioner. } \\
\text { a. } N \text { and } n \text {, unweighted number of respondents. } \\
\text { b. Odds ratios (ORS) are from univariate logistic regression analyses with other mental health service use as the reference group. } \\
\text { c. } n=498 \text { and } n=124 \text { for this variable. } \\
\text { d. } n=480 \text { and } n=123 \text { for this variable. }\end{array}$} \\
\hline
\end{tabular}

manage themselves. Cost was identified as a barrier by less than one in five people.

Non-service users did not report any use of complementary therapies. In addition, only $1.3 \%$ (s.e. $=0.3$ ) used self-management strategies, a significantly lower proportion than the Better Access service users $(17.9 \%$, s.e. $=5.6, \quad \mathrm{OR}=16.0,95 \%$ CI $5.4-47.2$, $P<0.001)$ or the other service user groups $(13.4 \%$, s.e. $=2.3$, $\mathrm{OR}=11.3,95 \%$ CI 5.4-23.5, $P<0.001)$.

\section{Discussion}

\section{Summary and interpretation of findings}

This study provides evidence regarding five sources of potential failure in the implementation of the Better Access initiative. First, the majority of Better Access service users had a 12-month ICD-10 mental disorder $(81.7 \%)$ or another indicator of treatment need $(11.5 \%)$. Prevalence of 12 -month disorder was higher than among service users generally $(58.7 \%$ in the 2007 National Survey of Mental Health and Wellbeing ${ }^{21}$ and $61.2 \%$ in the National Comorbidity Survey Replication in the USA) ${ }^{22}$ suggesting that referrals being made to allied health providers under Better Access are appropriate. The estimate of $6.8 \%$ without an indicator of need is similar to US estimates of $8.0 \%$ among service users generally. ${ }^{17}$ It is possible that the true figure for Better Access psychological services is even lower, as the 6.8\% may have included people with disorders not assessed by the 2007 National Survey of Mental Health and Wellbeing or indicators of treatment need not assessed by this study (e.g. simple phobias, psychotic disorders, personality disorders, or subthreshold symptoms of these disorders). Regardless, it has been suggested that a false-positive treatment rate of this magnitude is acceptable. $^{17}$

Second, Better Access users without 12-month ICD-10 affective or anxiety disorders were not more socioeconomically advantaged. They tended to include fewer people born in Australia and more older people, but these differences were not statistically significant, possibly because of small numbers. Better Access users with a disorder received a higher intensity of treatment than those without. They also tended to receive more psychoeducation, medication and psychotherapy; but again small numbers may have limited the possibility of finding these differences statistically significant. Taken together, these findings indicate that resources are not being inappropriately diverted from people with genuine need.

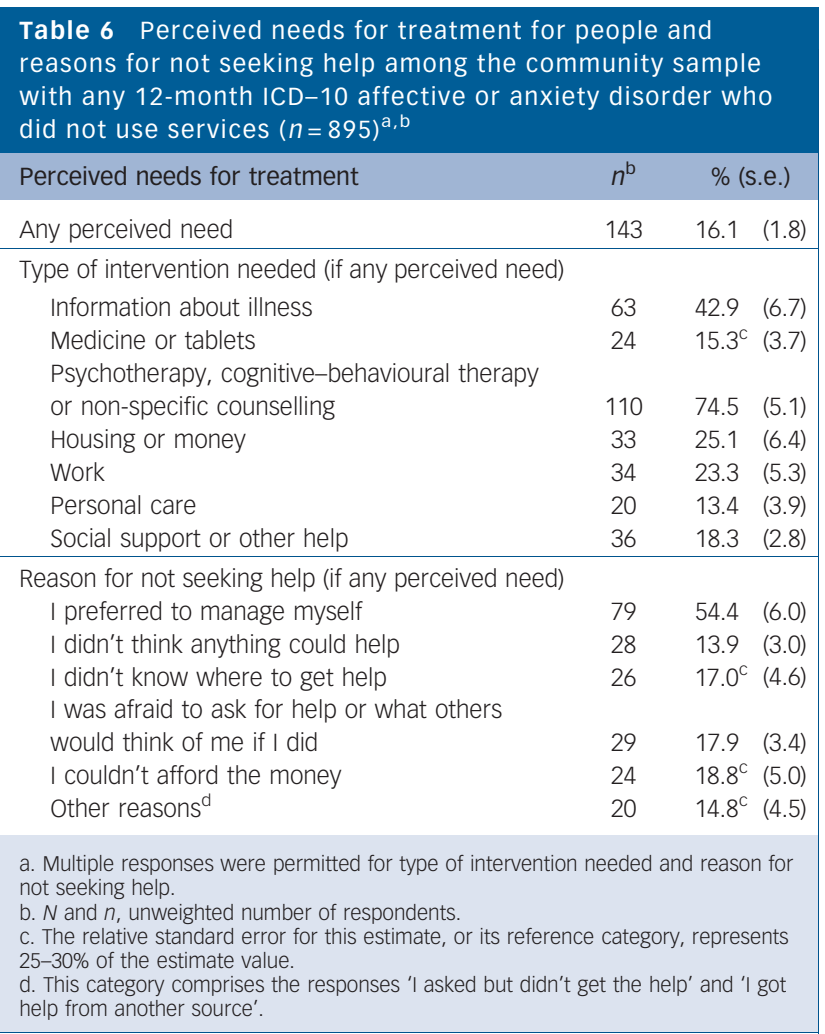


Third, our analyses among the community sample with 12-month ICD-10 affective or anxiety disorders did not indicate social inequalities in the use of Better Access psychological services, but did suggest that access may be better for some clinical groups than others. Even after controlling for severity of disorder, people with affective disorders were more likely to use Better Access psychological services than to not use services, whereas the reverse was true for people who had anxiety disorders that were not comorbid with affective disorders.

Evidence from population-based surveys and large studies of mental health service users suggest that there may be unequal access to psychological therapies and services provided by allied health professionals. Younger age, ${ }^{23}$ being separated ${ }^{23,24}$ or single, ${ }^{23}$ poorer education level, ${ }^{23}$ employment, ${ }^{25}$ higher income, ${ }^{25}$ and being Black or Asian ${ }^{26}$ have been found in some, but not all ${ }^{27}$ studies, to predict receipt of these treatments. In our study, type and severity of disorder were the only predictors of Better Access psychological service use compared with no service use. Geographical location, level of socioeconomic disadvantage and demographic factors were not significant predictors, after taking the effects of clinical factors into account. Moreover, there were no factors differentiating Better Access from other service use. Our findings suggest that Better Access is providing equitable access to people in need. However, our ability to draw comparisons with previous findings is limited by differences in the samples used for analysis (diagnostic groups, service users), study settings (general population, primary care) and in the definitions of the services being examined.

Better Access psychological services users more frequently reported receiving psychoeducation and non-specific counselling, but not CBT, than those who used other services. The CBT finding was contrary to expectation, given that Better Access was intended to promote the use of evidence-based therapies such as psychoeducation, CBT and interpersonal therapy. ${ }^{5}$ This finding may suggest that other services provide more CBT than expected, or that Better Access services provided greater levels of other therapies than expected. Alternately, it may reflect shortcomings in population mental health literacy relating to the correct labelling of specific interventions.

Fourth, although we were unable to derive precise estimates of the proportion of Better Access users whose first consultation with an allied health provider had occurred in the preceding year, our estimate of $62 \%$ is broadly consistent with findings from independent surveys of Australian psychologists who report that around $70 \%$ of their Better Access clients have not previously consulted a psychologist. ${ }^{28,29}$

Fifth, we found that $61.0 \%$ of people with 12 -month affective or anxiety disorders had not used services. Although this group could potentially benefit from Better Access, they reported low rates of perceived need for treatment $(16.1 \%)$ and did not seek treatment alternatives. Even among the $13.5 \%$ of non-service users with severe disorders, only a third perceived a need for treatment. Of those who did perceive a need for treatment, cost was identified as a barrier to receiving services by only one in five. Factors such as knowledge or beliefs about the availability and effectiveness of treatments, and stigma, appear to collectively have a stronger influence on perceptions of treatment need.

Levels of perceived need among non-service users (of whom $86.4 \%$ had anxiety disorders) were much lower than those previously reported from the 1997 National Survey of Mental Health and Wellbeing among people with full-threshold 12-month anxiety disorders, but similar to those with subthreshold anxiety disorders. ${ }^{30}$ This may be due to less strict operationalisation of diagnostic criteria in the 2007 National Survey of Mental Health and Wellbeing. ${ }^{12}$

\section{Threats to validity}

\section{Are the Better Access service use estimates valid?}

We estimated that 290140 adults are likely to have received Better Access psychological services from allied health professionals in the first year of the programme, but note that the survey measures of health service use, including use of Medicare-funded services, rely on unverified, retrospective self-report. We derived similar estimates of 295000 and 305000 from independently published analyses of Medicare claims data. ${ }^{5,31}$ The slightly lower surveybased estimate reflects the timing of the survey, which meant that not all respondents would have accrued a full year of opportunity to receive Better Access services, and because it was limited to people aged 16-85 years. We found good agreement with published Medicare data ${ }^{30}$ with respect to rates of Better Access treatment by age, gender and urbanicity. Comparisons by type of professional consulted suggest that the survey may overestimate rates of Better Access treatment by social workers or occupational therapists and underestimate those from psychologists. This may indicate shortcomings in the general public's ability to reliably identify the specific disciplines of mental health professionals, and may also reflect previous findings that counsellors are identified by more people as helpful for depression than psychologists. ${ }^{32}$ Overall, however, the comparisons lend support for the validity of the survey-based estimates of Better Access service use.

\section{Other methodological considerations}

Other potential limitations to the study require comment. First, the survey response rate of $60 \%$ may have introduced selection biases, however we do not know whether these may have affected participation rates among people with mental disorders. Second, the extent to which inferences about causality can be made is limited by the cross-sectional nature of the survey. ${ }^{11,12}$ Third, the survey did not ask respondents about use of Better Access services by name. However, as we do not know whether consumers would correctly identify the services they received as being part of Better Access, we consider that asking individuals about the professionals consulted and means of payment is the most accurate way to determine Better Access service use under survey conditions. As noted earlier, this method may have captured people receiving Medicare-subsidised allied health services provided under other programmes, however the number is considered negligible. Fourth, membership of service use groups was hierarchical and allocated anyone who reported receiving Medicare-subsidised allied health services to the Better Access group, even though they may have had multiple payers for one consultation, or had multiple payers across multiple consultations. Finally, as already noted, the relatively small numbers available for some comparisons of Better Access users may have limited the power of our study to detect some differences in sociodemographic profile and treatments received as statistically significant. Importantly, we followed standard best practice in reporting estimates from population surveys. We identified a small number of estimates with relative standard error percentages above the $25 \%$ threshold but in each of these cases the relative standard error percentage was not greater than $30 \%$, thus any limitations to reliability can be considered relatively small.

\section{Policy and research implications}

The high personal, social and economic costs associated with common mental disorders have led governments to implement major primary mental healthcare reforms such as Better Access and Improving Access to Psychological Therapies to improve 
access to psychological treatments. However, the potential for such programmes to create, or widen, existing disparities in mental health service use is of concern. ${ }^{4,7-9,33,34}$ Our findings suggest two implications for policy. First, although Better Access appears to be providing equitable access, people who have anxiety disorders that are not comorbid with affective disorders appear to be a group for whom detection and/or referral to Better Access psychological services could be improved. Second, a substantial number of people with affective or anxiety disorders, who could potentially benefit from Better Access services, did not use these or any other services. Non-service was not associated with socioeconomic or geographical factors, but perceived need for treatment in this group (even among those with severe disorders) was low. Mental literacy efforts may be more helpful in improving treatment rates among this group than increasing opportunities for access to treatment.

Our findings also have research implications. To reduce the burden of common mental disorders it is necessary to not only increase treatment coverage to populations in need, but also to ensure that evidence-based treatments are being delivered to those who gain access. ${ }^{35,36}$ At a clinical level, longitudinal studies of Better Access consumers are needed to examine the quality, outcomes and technical efficiency of the programme. At a population level, findings from the 1997 and 2007 National Survey of Mental Health and Wellbeing suggest that although overall rates of service use by people with affective and anxiety disorders did not change over the intervening decade, treatment by psychologists doubled, ${ }^{37}$ presumably due to Better Access and other reforms. ${ }^{38}$ Using epidemiological data from the 1997 National Survey of Mental Health and Wellbeing, coupled with evidence and expert opinion, Andrews and colleagues estimated the number of psychological services required to provide optimal treatment at optimal coverage to people with affective and anxiety disorders. ${ }^{35,39}$ Future analyses of the 2007 National Survey of Mental Health and Wellbeing may inform whether large-scale primary mental healthcare reforms such as Better Access can contribute to improvements in treatment quality and coverage for these disorders.

\section{Meredith G. Harris, MPH, Philip M. Burgess, PhD, The University of Queensland School of Population Health, Brisbane, Queensland; Jane E. Pirkis, PhD, The University of Melbourne, School of Population Health, Melbourne, Victoria; Tim N. Slade, PhD, University of New South Wales, National Drug and Alcohol Research Centre, Sydney, New South Wales; Harvey A. Whiteford, MPH, FRANZCP, The University of Queensland, School of Population Health, Brisbane, Queensland, Australia}

Correspondence: Meredith G. Harris, MPH, Queensland Centre for Mental Health Research, Locked Bag 500, Sumner Park BC, Queensland 4077, Australia. Email: harrism@qcmhr.uq.edu.au

First received 1 Oct 2009, final revision 20 Apr 2010, accepted 2 Jun 2010

\section{Funding}

The 2007 National Survey of Mental Health and Wellbeing was funded by the Australian Government Department of Health and Ageing, and conducted by the Australian Bureau of Statistics.

\section{References}

1 Andrews G, Sanderson K, Slade T, Issakidis C. Why does the burden of disease persist? Relating the burden of anxiety and depression to effectiveness of treatment. Bull World Health Organ 2000; 78: 446-54.

2 Andrews G, Henderson S, Hall W. Prevalence, comorbidity, disability and service utilisation. Overview of the Australian National Mental Health Survey. Br J Psychiatry 2001; 178: 145-53.
3 Littlefield L, Giese J. The genesis, implementation and impact of the Better Access mental health initiative introducing Medicare-funded psychology services. Clin Psychol 2008; 12: 42-9.

4 Clark D, Layard R, Smithies R, Richards D, Suckling R, Wright B. Improving access to psychological therapy: initial evaluation of two UK demonstration sites. Behav Res Ther 2009; 47: 910-20.

5 Whiteford HA, Doessel DP, Sheridan JS. Uptake of Medicare Benefits Schedule items by psychologists and other mental health practitioners. Clin Psychol 2008; 12: 50-6.

6 Medicare Australia. Medicare Item Reports. Australian Government, 2010 (http://www.medicareaustralia.gov.au/statistics/mbs_item.shtml).

7 Rosenberg S, Hickie IB, Mendoza J. National mental health reform: less talk, more action. Med J Aust 2009; 190: 193-5.

8 Hickie IB, McGorry PD. Increased access to evidence-based primary mental health care: will the implementation match the rhetoric? Med J Aust 2007; 187: $101-3$.

9 Crosbie D, Rosenberg S. Mental Health and the New Medicare Services: An Analysis of the First Six Months. Mental Health Council of Australia, 2007.

10 Dunbar JA, Hickie IB, Wakerman J, Reddy P. New money for mental health: will it make things better for rural and remote Australia? Med J Aust 2007; 186: $587-9$.

11 Australian Bureau of Statistics. 2007 National Survey of Mental Health and Wellbeing: Users' Guide (Cat. No. 4327.0). Australian Bureau of Statistics, 2009.

12 Slade T, Johnston A, Oakley Browne MA, Andrews G, Whiteford H. 2007 National Survey of Mental Health and Wellbeing: methods and key findings. Aust N Z J Psychiatry 2009; 43: 594-605.

13 Kessler RC, Üstun TB. The World Mental Health (WMH) Survey Initiative Version of the World Health Organization (WHO) Composite International Diagnostic Interview (CIDI). Int J Methods Psychiatr Res 2004; 13: 93-121.

14 World Health Organization. The ICD-10 Classification of Mental and Behavioural Disorders: Clinical Descriptions and Diagnostic Guidelines. WHO, 1992.

15 World Health Organization. WHODAS II Disability Assessment Schedule. WHO, 2001 (http://www.who.int/icidh/whodas/).

16 Kessler R, Mroczek, D. Final versions of our Non-specific Psychological Distress Scale. Memo dated March 10 1994. Survey Research Center for Social Research, University of Michigan, 1994.

17 Druss BG, Wang PS, Sampson NA, Olfson M, Pincus HA, Wells KB, et al. Understanding mental health treatment in persons without mental diagnoses: results from the National Comorbidity Survey Replication. Arch Gen Psychiatry 2007; 64: 1196-203.

18 Meadows G, Harvey C, Fossey E, Burgess P. Assessing perceived need for mental health care in a community survey: development of the Perceived Need for Care Questionnaire (PNCQ). Soc Psychiatry Psychiatr Epidemiol 2000; 35: 427-35

19 Australian Bureau of Statistics. Information Paper: An Introduction to Socio-Economic Indexes for Areas (SEIFA), 2006 (Cat. No. 2039.0). Australian Bureau of Statistics, 2008.

20 Australian Bureau of Statistics. 2007 National Survey of Mental Health and Wellbeing: Summary of Results (Cat. No. 4326.0). Australian Bureau of Statistics, 2008.

21 Slade $T$, Johnston $A$, Teesson $M$, Whiteford $H$, Burgess $P$, Pirkis J, et al. The Mental Health of Australians 2. Report on the 2007 National Survey of Mental Health and Wellbeing (Cat. No. 4327.0). Commonwealth of Australia, 2009.

22 Wang PS, Aguilar-Gaxiola S, Alonso J, Angermeyer MC, Borges G, Bromet EJ, et al. Use of mental health services for anxiety, mood, and substance disorders in 17 countries in the WHO world mental health surveys. Lancet 2007; 370: 841-50.

23 Kessler R, Demler O, Frank RG, Olfson M, Pincus HA, Walters EE, et al. Prevalence and treatment of mental disorders, 1990 to 2003. N Eng J Med 2005; 352: 2515-23.

24 Parslow R, Jorm A. Predictors of types of help provided to people using services for mental health problems: an analysis of the Australian National Survey of Mental Health and Wellbeing. Aust N Z J Psychiatry 2001; 35: 183-9.

25 Issakidis C, Andrews G. Who treats whom? An application of the Pathways to Care model in Australia. Aust N Z J Psychiatry 2006; 40: 74-86.

26 Raleigh VS, Irons R, Hawe E, Scobie S, Cook A, Reeves R, et al. Ethnic variations in the experiences of mental health service users in England. Results of a national patient survey programme. Br J Psychiatry 2007; 191: $304-12$

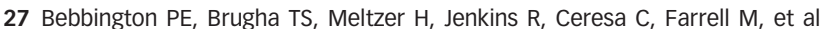
Neurotic disorders and the receipt of psychiatric treatment. Psychol Med 2000; 30: 1369-76. 
28 Giese J, Littlefield L, Mathews R. Survey of members providing Medicarefunded services under the Better Access initiative. InPsych: Bull Australian Psychol Soc 2008; 30: 36-7.

29 Forsyth C, Mathews R. Survey of members providing services under the Better Access and Better Outcomes initiatives. InPsych: Bull Australian Psychol Soc 2009; 31: 30-3.

30 Issakidis C, Andrews G. Service utilisation for anxiety in an Australian community sample. Soc Psychiatry Psychiatr Epidemiol 2002; 37: 153-63.

31 Australian Institute of Health and Welfare. Mental Health Services in Australia, 2006-07. Australian Institute of Health and Welfare, 2009.

32 Jorm AF, Christensen $\mathrm{H}$, Griffiths KM. The public's ability to recognize mental disorders and their beliefs about treatment: changes in Australia over 8 years. Aust N Z J Psychiatry 2006; 40: 36-41.

33 Department of Health. Improving Access to Psychological Therapies Implementation Plan: Equality Impact Assessment. Department of Health, 2008.
34 Ghosh P. Improving access to psychological therapies for all adults. Psychiatr Bull 2009; 33: 186-8.

35 Andrews G, Tolkien II Team. Tolkien II: A Needs-based, Costed Stepped-care Model for Mental Health Services. World Health Organization Collaborating Centre for Classification of Mental Health, 2007.

36 Whiteford HA. Depression in primary care: expanding the evidence base for diagnosis and treatment. Med J Aust 2008; 188: S101-2.

37 Burgess PM, Pirkis JE, Slade TN, Johnston AK, Meadows GN, Gunn JM. Service use for mental health problems: findings from the 2007 National Survey of Mental Health and Wellbeing. Aust N Z J Psychiatry 2009; 43: 615-23.

38 Whiteford $\mathrm{H}$, Groves A. Policy Implications of the 2007 National Survey of Mental Health and Wellbeing. Aust N Z J Psychiatry 2009; 43: 644-51.

39 Andrews G, Issakidis C, Sanderson K, Corry J, Lapsley H. Utilising survey data to inform public policy: comparison of the cost-effectiveness of treatment of ten mental disorders. Br J Psychiatry 2004; 184: 526-33.

\section{fallen \\ stars}

\section{Gérard de Nerval - the man who walked lobsters}

\section{Raymond Cavanaugh Jr}

Gérard de Nerval, a.k.a. Gerard Labrunie, was born in Paris in 1808. The son of a military doctor in Napoleon's Grande Armée, de Nerval's mother died shortly after his birth.

The young child was sent to a maternal relative in the French countryside, where he spent several years. De Nerval was eventually reunited with his father in Paris; however, he would occasionally return to that seemingly boundless landscape which had kindled his imagination.

Though de Nerval would produce his own creative works, he first made his mark at the age of 20 with a heralded translation of Goethe's epic Faust. The young man would undertake other translations, and he has been credited with introducing the poems of Heinrich Heine to French readers.

Concurrent with those endeavours, de Nerval joined a very bohemian peer group consisting of brilliant misfits who together pursued the artificial paradise of hashish.

Though one must be wary of attributing artistic composition to hashish, de Nerval clearly did manifest a strong drive for altered states of consciousness. He kept pursuing an exalted 'spirit world' as opposed to the material world he dismissed as a 'hovel and place of illrepute' before proclaiming: 'I am ashamed that God should see me here'.

As part of his preferred 'spirit world', de Nerval placed much significance on dreams, a priority which would later find emphasis in the Surrealist movement. Fellow Frenchmen, such as Marcel Proust, have felt his dreamy influence, and T. S. Eliot's groundbreaking Wasteland epic would include the second line of de Nerval's sonnet El Desdichado.

In 1841, de Nerval's world of spirits plunged into disarray when he suffered his first nervous breakdown. He embarked on Les Illuminés, a series of short novels on the theme of mental instability.

Around this time, de Nerval began to acquire quite a reputation for walking a pet lobster on a leash through the Palais Royal Gardens in Paris. 'I have a liking for lobsters', he declared. 'They are peaceful, serious creatures who know the secrets of the sea and don't bark'.

Though he provided a rather compelling explanation, the sight of de Nerval circling Paris with a pet lobster only added to suspicions of insanity. Beset by subsequent nervous breakdowns, his financial situation worsened; at one point he was 'accosted by the mayor for stealing from the lobster nets'.

On a morning in January 1855, de Nerval was found dangling beneath the splintery grate of a rooming house, having hanged himself with bondage wire snatched from an adjoining brothel. He left a note which read: 'Do not wait up for me this evening, for the night will be black and white'. 